\title{
A phase II study on the efficacy and safety of procedural analgesia with fentanyl buccal tablet in cancer patients for the placement of indwelling central venous access systems
}

\author{
R Bortolussi $^{1} \cdot$ P Zotti $^{2} \cdot$ M Matovic $^{3} \cdot$ A Morabito $^{3} \cdot$ C Bertuzzi $^{3} \cdot$ M Caserta $^{3}$. \\ F Fabiani $^{3}$ - A Fracasso ${ }^{3}$ - C Santantonio ${ }^{3}$ - C Zanier $^{3}$ - A Roscetti ${ }^{3}$ - J Polesel ${ }^{4}$. \\ D Gussetti $^{5} \cdot$ S Bedin $^{3} \cdot$ AM Colussi $^{5} \cdot$ D Fantin $^{3}$
}

Received: 25 March 2015 / Accepted: 3 September 2015 /Published online: 16 September 2015

(C) The Author(s) 2015. This article is published with open access at Springerlink.com

\begin{abstract}
Background Pain in cancer patients is often related to oncologic therapies and diagnostic procedures. The placement of fully implantable venous access systems is a very common procedure in oncology patients. Local anaesthesia is the method most commonly used to overcome pain related to this surgical procedure, but the local anaesthetic may be unable to completely eradicate all pain. This study investigates the effectiveness and safety of fentanyl buccal tablet (FBT), administered by OraVescent ${ }^{\mathbb{R}}$ technology, in reducing procedural pain related to the placement of indwelling central venous access systems (Ports) in opioid-naïve cancer patients.

Methods Inpatients who required an indwelling vascular access (Port) were preoperatively assessed with a selfassessment questionnaire on anxiety and pain. A $100 \mu \mathrm{g}$ FBT was administered 10 min before preparation of the operating field. A self-assessment scale for pain experienced during the procedure was administered at the end of the procedure. Vital signs and the presence of any side effects or
\end{abstract}

R Bortolussi

rbortolussi@cro.it

1 Pain Therapy and Palliative Care Unit, CRO Aviano National Cancer Institute, Aviano, Italy

2 Psycho-Oncology Unit, CRO Aviano National Cancer Institute, Aviano, Italy

3 Anaesthesia and Intensive Care Unit, CRO Aviano National Cancer Institute, Aviano, Italy

4 Epidemiology and Biostatistics Dept, CRO Aviano National Cancer Institute, Aviano, Italy

5 Clinical Trials Office, Scientific Directorate, CRO Aviano National Cancer Institute, Aviano, Italy bothersome symptoms were monitored during the procedure, at the end, and $4 \mathrm{~h}$ later.

Results From October 2012 to June 2014, 65 patients were enrolled in the study. A total of 61 (93.9\%) patients perceived no or a little pain during the procedure. Four patients $(6.2 \%)$ reported a lot of pain. No patient reported very severe pain. This data is significant in terms of the lower than expected presence of pain (Fisher test $p=0.0018$ ) as assessed in our previous experience without procedural analgesia. The most common side effects of FBT was drowsiness, experienced by 28 patients at the end of the procedure (43.1\%), significantly reduced $(p<0.01)$ to 8 patients after $4 \mathrm{~h}(12.5 \%)$. Nausea was present in 6 cases at the end of the procedure (9.2\%) and in 7 cases $4 \mathrm{~h}$ later $(10.9 \%)$. Vomiting was present in 3 cases at the end $(4.7 \%)$ and in 2 other patients after $4 \mathrm{~h}(7.8 \%)$. No significant change of vital parameters was observed between the baseline and the subsequent measurements in all patients studied.

Conclusions The significant improvement in the number of patients experiencing little or no pain, accompanied by a lower number of non-severe side effects, suggests that FBT is a valid, practical and safe method of procedural analgesia. It will be necessary to perform further studies, taking into account the need for standard antiemetic pre-medication to minimise the incidence of nausea and vomiting.

\section{Keywords Pain $\cdot$ Cancer $\cdot$ Anaesthesia}

\section{Introduction}

During the course of their illness, cancer patients often experience pain that can be proportionately greater in the more advanced stages of the disease. Of the various types of pain 
perceived, one particular form is procedural pain. Invasive endoscopic, radiological and surgical diagnostic procedures are mandatory steps in the diagnosis and therapy and can be associated with pain that affects the patient's quality of life [1]. The recommendation to prepare treatment protocols in order to eliminate procedural pain has been expressed in wellknown guidelines [2]. The need to implement protocols to achieve this aim is considered to be particularly important when procedures involve cancer patients [3]. However, these recommendations are frequently overlooked and procedural pain is often not treated adequately [4]. In specific clinical fields, such as paediatrics, the need to improve prevention and treatment of procedural pain has recently encouraged research in this care problem [5, 6]. For digestive endoscopy, there are guidelines written by the major scientific societies which recommend the gold standards, the best procedures and the necessary training for medical professionals [7]. However, there are no recent articles on procedural pain in the adult oncology patient. The reason for this lies not only in the limited awareness about this topic of healthcare professionals, but also in the objective difficulties that can be present in the administration of procedural sedation and analgesia. The placement of central venous catheters in cancer patients before they start chemotherapy $(\mathrm{CT})$ is a common procedure $[8,9]$, and central venous access is usually obtained by catheterization of the superior vena cava. The high blood flow and large calibre of this blood vessel alone are able to neutralise the phlebotic effect of continuous infusions of solutions and chemotherapy and to allow the placement of venous catheters for long periods [10]. In short, central venous accesses can be classified as external catheters or fully implantable systems. The latter are constituted by a reservoir (Port) connected to a venous catheter whose tip is either open or closed by a valve. The catheter is usually positioned in the vein using a percutaneous technique. An incision is made in the skin and the Port is placed inside a pocket of subcutaneous tissue in the subclavicular area, on the same side of the venipuncture site. The connection between the catheter and the Port is performed by tunnelling the catheter from the venipuncture site to the subcutaneous pocket. Unlike external systems, implantable systems preserve body image and are therefore better accepted by patients from a cosmetic and psychological point of view. In addition, they present the advantage of minimal interference with daily living activities and make it possible, between treatments, to maintain normal personal hygiene (e.g. baths and showers). The positioning of a fully implantable venous access system therefore constitutes a minor surgical procedure, performed by a team including a surgeon or an anaesthesiologist who is optionally assisted by a second, and a nurse. Local anaesthesia is the method most commonly used to overcome pain related to the venipuncture, skin incision, passage of the tunneller for the catheter and the detachment of the subcutaneous tissue required to house the Port. This makes it possible to have a cooperative patient during the vein identification and catheter introduction phases, which are facilitated by breathing and apnoea as ordered by the physician performing the procedure. However, difficulties regarding pain-related fear are often experienced during the injection of the local anaesthetic and difficulties in positioning the implants related to the pain caused by manoeuvres that cannot always be perfectly controlled by local anaesthetic infiltration (such as the catheter tunnelling phase). This entails reduced patient cooperation and greater procedure-related stress. The local anaesthetic may be unable to completely abolish all pain, as its infiltration can itself cause severe burning before the induction of anaesthesia, as well as the pain stimulus caused in the pectoral muscle fascia during detachment. As regards the central venous incannulation procedure for the positioning of the Port, patients often present compromised peripheral veins, making it difficult to identify a peripheral vein for the administration of the procedural sedation and analgesia. In other cases, the patient's fear of the pain related to the venipuncture is another limiting factor. However, the intravenous route is the route of election because of its rapid effect and the possibility of using drugs with a short half-life, both essential characteristics for ambulatory day hospital procedures with a mean duration of 30-40 min, such as those for central venous incannulation. Conventional oral drugs such as benzodiazepines and short-release opioids have a latency of more than $30 \mathrm{~min}$ and duration of action of between 4 and $8 \mathrm{~h}$, and therefore, their characteristics are not ideal for use in the procedure in question. One potent opioid frequently used for procedural sedation and analgesia is fentanyl. This opioid is 100 times more potent than morphine and is highly lipophilic and therefore crosses the blood-brain barrier rapidly. These characteristics have made it one of the most common drugs used in modern surgical anaesthesia and it has also been extensively used in other clinical settings. It has been recognised as being efficacious and manageable in radiological procedural sedation and analgesia, where it is usually used at a dose of $1 \mu \mathrm{g} / \mathrm{kg}$ i.v. either alone or in combination with midazolam $[11,12]$. It has also been used with similarly demonstrated efficacy and manageability when administered intravenously at doses of between 1 and $2 \mu \mathrm{g} / \mathrm{kg}$ in the treatment of prehospital pain management [13]. It has also recently been used to treat procedural pain associated with venipuncture [14].

\section{Description of the investigational medicinal product}

For some years now, fentanyl has been available as a transmucosal formulation. This method of administration was devised to treat breakthrough cancer pain, i.e. the acute and sudden pain that presents over background pain in patients on long-term opioid therapy. It has already been used to produce procedural sedation and analgesia in cancer 
patients eligible for radiotherapy [15]. Fentanyl buccal tablet (FBT) is a more recent formulation of transmucosal fentanyl citrate. It uses OraVescent ${ }^{\circledR}$ technology to further increase the rate and extent of absorption of fentanyl provided by its predecessor compound, oral transmucosal fentanyl citrate (OTFC). It improves dissolution and passage of the drug into the circulation by selectively modifying the local $\mathrm{pH}$ and permeability at the mucosal wall. An initial decrease in $\mathrm{pH}$ promotes the action of the soluble, ionised, hydrophilic form of fentanyl, while a subsequent, gradual increase in $\mathrm{pH}$ promotes the more absorbable, nonionized, lipophilic form. Fentanyl is absorbed immediately after the administration of the tablet with $65 \%$ bioavailability, making it possible to induce rapid analgesia after 5 to $15 \mathrm{~min}$, with a peak in the effects within $2 \mathrm{~h}$ and a half-life that does not exceed $4 \mathrm{~h}$. One $100-\mu \mathrm{g}$ tablet therefore releases a dose equivalent to $65 \mu \mathrm{g}$ administered intravenously [16, 17]. The drug has been approved for use in patients pre-treated with daily opioid doses equal to $60 \mathrm{mg}$ of oral morphine sulphate. The aim of this is to minimise the opioid side effects such as nausea, vomiting, drowsiness, pruritus, mild hypotension and respiratory depression. Given its characteristics, this formulation of fentanyl appeared to us to be ideal for producing basic procedural analgesia prior to the administration of local anaesthesia in Port-positioning candidates. The buccal transmucosal administration route does not require the use of a peripheral venous access and it is similar, in terms of analgesic efficacy and latency, to an intravenous injection, which is the most common route of administration of the procedural analgesics indicated previously.

\section{Aim of the study}

The primary aim of the study was to evaluate FBT's capacity to reduce procedural pain related to the placement of an indwelling central venous access system (Ports) in opioid-naïve cancer patients.

The study's secondary objectives were (i) to evaluate the safety of FBT administration in opioid-naïve patients in a hospital setting and (ii) to evaluate the interference of pain-related anxiety and fear experienced by patients before the procedure in terms of their perception of pain.

\section{Study design}

From October 2012 till June 2014, all consecutive inpatient candidates for indwelling vascular access (Port) positioning for the administration of chemotherapy for histologically diagnosed cancer were assessed for eligibility. Before entering the operating theatre (study time $=\mathrm{T} 0$ ), those patients who met the inclusion and exclusion criteria and had given their informed consent to participate in the above study were given a self-assessment questionnaire on anxiety (State-Trait Anxiety Inventory Y-1 form) [18] and pain expectation; replies were on a 4-point Likert scale: 'not at all', 'a little', 'quite a lot' or 'extremely painful'. Patients with a history of anaesthesia-related nausea were treated with one metoclopramide tablet $60 \mathrm{~min}$ before the procedure. Patients received one buccal tablet of FBT. $100 \mu \mathrm{g} 10 \mathrm{~min}$ before the preparation of the operating field, and at the same time, their vital parameters were monitored (respiratory rate, blood pressure and peripheral arterial oxygenation). Self-assessment scales for the pain experienced during the procedure and a Visual Numeric Rating Scale 0-10 (VNRS 0-10) were administered at the end of the procedure (study time $=\mathrm{T} 1$ ). Vital parameters were monitored throughout the procedure. Patients presenting pain that was self-assessed as significant during the procedure (a lot of pain or extremely painful VNRS $\geq 5$ ) were provided with rescue therapy of one ampoule containing $30 \mathrm{mg}$ of ketorolac for sub-lingual administration or tramadol 20 drops for patients with a positive history of reactions to NSAIDs and ASA. Before the patients were sent to the Radiology department for a follow-up chest X-ray, the physician performing the procedure checked their vital signs and the presence of any side effects or bothersome symptoms (T1). A further closure assessment with the monitoring of vital parameters, side effects and bothersome symptoms was performed $4 \mathrm{~h}$ after the Port implantation (study time $=\mathrm{T} 2$ ). A pure opioid antagonist (naxolone) was readily available for use throughout the procedure period to control any severe side effects related to FBT administration.

\section{Inclusion criteria}

The inclusion criteria are as follows:

(1) Patients aged over 18 years.

(2) Histologically confirmed cancer diagnosis.

(3) Indication for the placement of the indwelling central venous catheter connected to the subcutaneous Port as an inpatient.

(4) Unimpaired cognitive capacity.

(5) Written informed consent.

\section{Exclusion criteria}

The exclusion criteria are as follows:

(1) Xerostomy or any other condition that does not make it possible to take effervescent tablets.

(2) Patients already being treated with opioids for chronic cancer pain. 
(3) A documented history of significant nausea and vomiting related to the administration of opioids.

(4) A history of intolerance to intravenous fentanyl or FBT.

(5) Severe or moderate chronic respiratory insufficiency.

(6) Patients with renal or hepatic function impairment.

\section{Statistical considerations}

Central venous catheterization is a common procedure considered to be at risk in terms of pain, anxiety and discomfort [19]. However, there is no data available in literature on the prevalence of pain during the placement of an indwelling venous system or Port. In 2010 and 2011, a total of 1152 Ports were positioned in the Anaesthesiology and Intensive Care Unit of this Institute. The pain perceived by patients during the procedure was routinely assessed using a 4-point Likert scale and VNRS $0-10$. The pain was judged as being no pain in 242 (21\%) patients, a little pain in $760(66 \%)$ patients, a lot of pain in $92(8 \%)$ patients and extremely painful in $57(5 \%)$ patients (unpublished data). With the procedural analgesia administered using the study product, which preceded the injection of the local anaesthetic by at least $15 \mathrm{~min}$, we considered a possible reduction to $5 \%$ in the number of patients experiencing a lot of pain or extreme pain. No placebo-controlled study was conducted to avoid ethical issues and further discomfort for patients as, being cancer sufferers, they are likely to experience pain during the course of their illness. The study caseload was calculated using Simon's method [20]. Considering an optimum two-stage design for phase II studies, with an expected $p_{1}-p_{0}$ difference of $10 \%$ between the percentage of patients expressing no or little pain during positioning with standard local anaesthesia $\left(p_{0}=85 \%\right)$ and that with the administration of FBT $\left(p_{1}=95 \%\right)$, and setting the probability of error at $\alpha=0.05$ and $\beta=0.20$, the number of patients needed for stage one was 13 . The study was to be ended if fewer than 11 patients with none or mild pain were enrolled in stage one. Otherwise, the study would have had to continue up to the enrolment of a total of 65 patients. The efficacy of FBT administration was also evaluated by stratifying analysis according to the patient's anxiety state before the procedure.

\section{Results}

The study was conducted from October 2012 till 30 June 2014. During this period, a total of 513 patients were candidates for the placement of venous Port as inpatients at this institute. Of these, $65(12.6 \%)$ met the study inclusion criteria, gave their informed consent and were enrolled in the study. The sociodemographic characteristics of the patients enrolled in the study are described in Table 1 . The majority of cases were women and the median age was 56 years (range 19-76 years).

A total of $61(93.9 \%)$ patients perceived none or a little pain during the procedure. Four patients $(6.2 \%)$ reported a lot of pain (Table 2). No patient reported extreme pain. We found a statistically significant reduction in pain perception compared to pain assessment of patients not treated with FBT in the years 2010-2011 (Fisher test $p=0.0018$ ). The rescue drugs identified for severe pain were never administered as no patient reported extreme pain (VNRS $\geq 8$ ) during the procedure (as reported at the final interview), while those who experienced a lot of pain $(\mathrm{VNRS} \geq 5<8)$ preferred not to take additional drugs. The pain expectation prior to the intervention and the pre-procedure level of anxiety in patients, measured using the STAI-Y test, did not significantly affect the perception of pain. Similarly, there were no statistically significant inequalities for gender, age group $(<55$ or $\geq 55$ years $)$ or level of education which were investigated using the preliminary interview (Table 2). The side effects of FBT administration are described in Table 3. Drowsiness was experienced by 28 patients at $\mathrm{T} 1(43.1 \%)$ and dropped significantly $(p<0.01)$ to 8 patients at T2 $(12.5 \%)$. Another commonly experienced symptom was vertigo, which was present in 19 cases at T1 $(29.2 \%)$ and dropped significantly $(p<0.02)$ to 8 cases $(12.5 \%)$ at $\mathrm{T} 2$. None of the patients described these effects as being bothersome. Nausea was present in 6 cases at T1 (9.2\%) and in 7 cases at T2 (10.9\%). Vomiting was present in 3 cases at $\mathrm{T} 1(4.7 \%)$ and in 2 other patients at T2 (7.8\%). No significant change was observed between the

Table 1 Sociodemographic and clinical characteristics of 65 patients

\begin{tabular}{lll}
\hline Characteristics & Number & Percentage \\
\hline Gender & & \\
$\quad$ Male & 19 & 29.2 \\
$\quad$ Female & 46 & 70.8 \\
Age (years) & & \\
$<45$ & 18 & 27.7 \\
$45-54$ & 12 & 18.5 \\
$55-64$ & 21 & 32.3 \\
$\geq 65$ & 14 & 21.5 \\
Education (years) & & \\
$<9$ & 25 & 38.5 \\
$9-13$ & 34 & 52.3 \\
$\geq 14$ & 6 & 9.2 \\
Occupation & & \\
White collar & 15 & 23.1 \\
Blue collar & 6 & 9.2 \\
Retired/housewife & 30 & 46.3 \\
Other & 14 & 21.5 \\
\hline
\end{tabular}


Table 2 Perception of pain during central venous catheter (CVC) positioning

\begin{tabular}{|c|c|c|c|c|c|c|c|}
\hline & \multicolumn{6}{|c|}{ Did you experience pain during the CVC positioning? } & \multirow{3}{*}{$\begin{array}{l}\text { VNRS (0-10) } \\
\text { Mean (SD) }\end{array}$} \\
\hline & \multicolumn{2}{|c|}{ No pain } & \multicolumn{2}{|c|}{ Little pain } & \multicolumn{2}{|c|}{ A lot of pain } & \\
\hline & $n$ & $\%$ & $n$ & $\%$ & $n$ & $\%$ & \\
\hline Overall & 33 & $(50.8)$ & 28 & $(43.1)$ & 4 & $(6.2)$ & $2.03(1.84)$ \\
\hline \multicolumn{8}{|l|}{ Gender } \\
\hline Male & 9 & $(47.4)$ & 7 & $(36.8)$ & 3 & $(15.8)$ & $2.50(2.28)$ \\
\hline \multirow[t]{2}{*}{ Female } & 24 & $(52.2)$ & 21 & $(45.7)$ & 1 & $(2.2)$ & $1.85(1.63)$ \\
\hline & \multicolumn{4}{|c|}{ Fisher test; $p=0.14$} & & & ANOVA; $p=0.21$ \\
\hline \multicolumn{8}{|l|}{ Age (years) } \\
\hline$<55$ & 14 & $(46.7)$ & 12 & $(40.0)$ & 4 & $(13.3)$ & $2.21(2.24)$ \\
\hline \multirow[t]{2}{*}{$\geq 55$} & 19 & $(54.3)$ & 16 & $(45.7)$ & 0 & $(0.0)$ & $1.89(1.45)$ \\
\hline & \multicolumn{4}{|c|}{ Fisher test; $p=0.10$} & & & ANOVA; $p=0.49$ \\
\hline \multicolumn{8}{|l|}{ Education (years) } \\
\hline$<9$ & 16 & $(64.0)$ & 9 & $(36.0)$ & 0 & $(0.0)$ & $1.80(1.58)$ \\
\hline $9-13$ & 15 & $(44.1)$ & 15 & $(44.1)$ & 4 & $(11.8)$ & $2.18(2.05)$ \\
\hline \multirow[t]{2}{*}{$\geq 14$} & 2 & $(33.3)$ & 4 & $(66.7)$ & 0 & $(0.0)$ & $2.17(1.84)$ \\
\hline & \multicolumn{4}{|c|}{ Fisher test; $p=0.23$} & & & ANOVA; $p=0.73$ \\
\hline \multicolumn{8}{|c|}{ Do you expect to experience pain during the CVC positioning? } \\
\hline No pain & 4 & $(80.0)$ & 1 & $(20.0)$ & 0 & $(0.0)$ & $1.20(1.10)$ \\
\hline Little pain & 24 & $(54.6)$ & 16 & $(36.4)$ & 4 & $(9.1)$ & $1.91(1.96)$ \\
\hline \multirow[t]{2}{*}{ A lot of pain/Extremely painful } & 5 & $(31.2)$ & 11 & $(68.8)$ & 0 & $(0.0)$ & $2.63(1.59)$ \\
\hline & \multicolumn{4}{|c|}{ Fisher test; $p=0.14$} & & & ANOVA; $p=0.24$ \\
\hline \multicolumn{8}{|c|}{ Anxiety before CVC positioning (STAI-Y) ${ }^{\mathrm{a}}$} \\
\hline Low & 7 & $(70.0)$ & 3 & $(30.0)$ & 0 & $(0.0)$ & $1.10(1.37)$ \\
\hline Medium & 24 & $(46.2)$ & 24 & $(46.2)$ & 4 & (7.7) & $2.24(1.90)$ \\
\hline \multirow[t]{2}{*}{ High } & 2 & $(66.7)$ & 1 & $(33.3)$ & 0 & $(0.0)$ & $1.67(1.53)$ \\
\hline & \multicolumn{4}{|c|}{ Fisher test; $p=0.63$} & & & ANOVA $; p=0.19$ \\
\hline
\end{tabular}

${ }^{\text {a }}$ Defined as lower or higher than the mean value $\pm \mathrm{SD}$, accounting for gender and age

baseline and subsequent measurements of vital parameters (blood pressure, peripheral blood $\mathrm{O}_{2}$ saturation by pulse oximetry and respiratory rate) monitored during the procedure and checked at $\mathrm{T} 1$ and $\mathrm{T} 2$.

Table 3 Frequency of side effects following central venous catheter positioning

\begin{tabular}{|c|c|c|c|c|c|}
\hline \multirow[t]{2}{*}{ Side effects } & \multicolumn{2}{|c|}{$\mathrm{T} 1(n=64)$} & \multicolumn{2}{|c|}{$\mathrm{T} 2(n=64)$} & \multirow[t]{2}{*}{$p$ value } \\
\hline & $n$ & $\%$ & $n$ & $\%$ & \\
\hline Dizziness & 28 & $(43.1)$ & 8 & (12.5) & $<0.01$ \\
\hline Nausea & 6 & $(9.2)$ & 7 & (10.9) & 0.75 \\
\hline Vomiting & 3 & (4.7) & 5 & $(7.8)$ & 0.47 \\
\hline Vertigo & 19 & (29.2) & 8 & (12.5) & 0.02 \\
\hline Other & 4 & $(6.2)$ & 3 & (4.7) & 0.71 \\
\hline
\end{tabular}

\section{Discussion}

Procedural analgesia in adult patients has not been extensively studied in recent literature. We found only one recent study performed with oncologic patients which investigated the safety and efficacy of methoxyflurane for procedural pain of bone marrow biopsy [21]. One recent study on procedural sedation and analgesia in the emergency department, where most procedures concerned fractures and dislocations, reported that midazolam is the most commonly used drug, frequently in combination with morphine [22]. Central venous incannulation procedure with placement of a Port is certainly far less invasive than the reduction of a fracture, and a midazolam-morphine combination would not have been the most appropriate analgesia option. Continuous remifentanil infusion at various doses between $0.025,0.005$ and $0.075 \mu \mathrm{g} / \mathrm{kg} / \mathrm{min}$ has been suggested for procedural sedation and analgesia for Ports and long-term venous catheters 
positioning and removal [23]. The drug has been proven to be an efficacious analgesic at all three doses, but is burdened by the risk of excessive sedation and respiratory and cardiovascular depression at higher doses. Continuous remifentanil infusion however requires the identification of a venous access prior to the procedure and close vital parameter monitoring. A study on procedural sedation and analgesia with fentanyl at a dose of $2 \mu \mathrm{g} / \mathrm{kg}$ versus placebo for central venous incannulation showed a significant level of pain reduction. [14]. This study considered short-term catheters, which are less invasive in terms of placement technique; however, the procedures are conducted using anatomical markers for vein identification.

This usually involves greater difficulty and a higher number of skin and vein punctures, with a higher failure rate than the ultrasound-guided incannulation technique we used. The precision and speed of ultrasound-guided puncture can be considered as a first factor for reducing patient discomfort and anxiety. Indeed, the ultrasound-guided technique allowed us to complete the procedure with little or no pain in $87 \%$ of the cases treated with anaesthesia by local infiltration alone prior to the start of the study. We believed that such a high number of procedures with low patient discomfort could be improved, by identifying a simple-to-administer premedication that does not require venipuncture and that is safe for the patient. The main feature of procedural analgesia for Port positioning is conscious sedation since it allows patient collaboration in the control of breathing, apnoea and the Valsalva manoeuvre. FBT pre-medication ensures this. The percentage of patients with no or little procedural pain was around $93.9 \%$ when local anaesthesia was associated with pre-medication with FBT. FBT is not currently approved for administration in opioid-naïve patients; however, its safety proved to be very high, without alterations in baseline parameters throughout the observation period of $4 \mathrm{~h}$ subsequent to administration. Amongst the various side effects, dizziness presented in a significant number of patients at T1 (43.1\%) but was never considered bothersome or unpleasant for the patient and was significantly reduced at T2 (12.5\%), corresponding to the $4 \mathrm{~h}$ following the end of the pharmacological effect of FBT. Vertigo, the second most common side effect, was also judged not to be bothersome or uncomfortable. Nausea and vomiting were less common but more uncomfortable, and unlike other side effects, they increased, albeit in a nonsignificant manner, at $\mathrm{T} 2$ compared to $\mathrm{T} 1$. In connection with this, it is important to note that all patients complaining of nausea following anaesthesia had been pre-medicated on the ward with $10 \mathrm{mg}$ of oral metoclopramide at the discretion of the anaesthesiologist who collected the consent. These patients did not present nausea and vomiting at $\mathrm{T} 1$ and $\mathrm{T} 2$ checks, which suggests that antiemetic pre-medication should always accompany the use of FBT as a procedural analgesic in opioid-naïve patients. The pre-procedure anxiety and pain expectation study showed that they did not have any influence on the perception of pain itself and they are not, therefore, useful, in our opinion, for selecting potential patients at risk of procedural pain.

\section{Conclusions}

The significant improvement in the number of patients experiencing no or little pain in procedural analgesia for central venous incannulation and the placement of a Port performed with FBT pre-medication and local anaesthesia infiltration compared to the standard technique performed with local infiltration anaesthesia alone, that was the technical standard of our procedure performed in the years prior to the study, was accompanied by a lower number of non-severe side effects. We believe that this suggests using FBT as a valid, practical and safe method of procedural analgesia. It is nevertheless necessary to perform further studies taking into account the need for standard antiemetic pre-medication to minimise the incidence of nausea and vomiting that appear to be limiting factors to the performance of procedural analgesia with FBT in opioid-naïve patients.

Conflict of interest The authors declare that they have no competing interests. None of the authors has a financial relationship with any organisation for which this research would present a conflict of interest. The data were in the hands of the authors at all times and are available to the journal upon request.

Open Access This article is distributed under the terms of the Creative Commons Attribution-NonCommercial 4.0 International License (http:// creativecommons.org/licenses/by-nc/4.0/), which permits any noncommercial use, distribution, and reproduction in any medium, provided you give appropriate credit to the original author(s) and the source, provide a link to the Creative Commons license, and indicate if changes were made.

\section{References}

1. Gavaruzzi T, Carnaghi A, Lotto L, Rumiati R, Meggiato T, Polato F, De Lazzari F (2010) Recalling pain experienced during a colonoscopy: pain expectation and variability. $\mathrm{Br} \mathrm{J}$ Health Psychol 15(Pt 2):253-264 Epub 2009 Jul 3

2. Agency for Health Care Policy and Research (1992). Acute pain management: operative or medical procedures and trauma. Clinical practice guidelines number 1 (AHCPR publications 92-0032) Rockville, MD: U.S. Department of Health and Human Services

3. Miaskowski C., Clearly J., Burney R., Coyne P., Finley R., Foster R. et al. (2005). Guidelines for the management of cancer pain in adults and children (APS Clinical Practice Guidelines Series, $\mathrm{N}^{\circ} 3$ ) Glenview IL: American pain Society

4. Puntillo KA, Wild LR, Moris AB, Stanik-Hutt J, Thompson CL, White C (2002) Practice and predictors of analgesic interventions for adults undergoing painful procedures. Am J Crit Care 11:415429 
5. Pò C, Benini F, Sainati L, Farina MI, Cesaro S, Agosto C (2012) The management of procedural pain at The Italian Centers of Pediatric Hematology-Oncology: state-of-the-art and future directions. Support Care Cancer 20(10):2407-2414

6. Kato Y, Maeda M, Aoki Y, et al (2014) Pain management during bone marrow aspiration and biopsy in pediatric cancer patients. Pediatr Int 56(3):354-359

7. Lee TH, Lee CK (2014) Endoscopic sedation: from training to performance. Clin Endosc 47:141-150

8. Feliciano DV, Mattox KL, Graham JM, et al. (1979) Major complications of percutaneous subclavian vein catheters. Am J Surg 138:869-874

9. De Cicco M, Bortolussi R, Fantin D, Matovic M, Fracasso A, Fabiani F, Santantonio C (2002) Supportive therapy of elderly cancer patients. Crit Rev Oncol Hematol 42(2):189-211

10. De Cicco M, Matovic M, Balestreri L, Steffan A, Pacenzia R, Malafronte M, Fantin D, Bertuzzi CA, Fabiani F, Morassut S, Bidoli E, Veronesi A (2009) Early and short term acenocumarine or dalteparin for the prevention of central vein catheter-related thrombosis in cancer patients: a randomized controlled study based on serial venographies. Ann Oncol 20(12):1936-1942

11. Tae-Hoon K (2006) Safety and effectiveness of moderate sedation for radiologic non-vascular intervention. Korean J Radiol 7:125130

12. Skehan JS, Malone DE, Buckley N, Masumoto S, Rawlinson J, Ting G, Graham D, Alexander J, Somerss S, Stevenson GW (2000) Sedation and analgesia in adult patients: evaluations of a staged-dose system based on body weight for use in abdominal interventional radiology. Radiology 216:653-659

13. Kanowitz A, Thomas M, Kanowitz EM, William W, Van Buskirk K (2006) Safety and effectiveness of fentanyl administration for prehospital pain management. Prehosp Emerg Care 10:1-7
14. Samantary A, Hanumantha RM (2014) Effects of fentanyl on procedural pain and discomfort associated with central venous catheter insertion: a prospective, randomized double-blind, placebo controlled trial. Indian J Crit Care Med 18(7):421-426

15. Proud C (2007) The use of oral transmucosal fentanyl citrate during high-dose-rate gynecologic brachytherapy. Clin J Oncol Nurs 11(4):561-567

16. Streisand JB, Varvel JR, Stanski DR, Le Maire L, Ashburn MA, Hague BI, et al. (1991) Absorption and bioavailability of oral transmucosal fentanyl citrate. Anesthesiology 75:223-229

17. Darwish M, Hamend E, Messina J (2010) Fentanyl buccal tablet for the treatment of breakthrough pain: pharmacokinetics of buccal mucosa delivery and clinical efficacy. Perspect Medicin Chem 4: $11-21$

18. Spielberger CD (1989) State-trait anxiety inventory: bibliography, 2nd edn. Consulting Psychologists Press, Palo Alto

19. Morrison RS, Ahronheim JC, Morrison JR, Darling E, Baskin SA, Morris J, et al. (1998) Pain and discomfort associated with common hospital procedures and experiences. J Pain Symptom Manag 15: 91-101fc

20. Simon R (1989) Optimal two-stage design for phase II clinical trials. Control Clin Trials 10(1):1-10

21. Spruyt O, Westerman D, Miner A, Bressel M, Wein S (2014) A randomized, double blind, placebo-controlled study to assess the safety and efficacy of methoxyflurane for procedural pain of a bone marrow biopsy. BMJ Support Palliat Care 4(4):342-348

22. Elkhodair SM, Baker EC, Glasebrook WR, Pott JD, Freund Y, Harris TR. (2014) Emergency department procedural sedation: the London experience. Eur J Emerg Med 12 (Epub ahead of print)

23. Burlacu CL, McKeating K, McShane AJ (2011) Remifentanil for the insertion and removal of long term central venous access during monitored anesthesia care. J Clin Anesth 23(4):286-291 